\title{
Anatomical and Centroid Point Variation in Cephalometrics
}

\section{Michael John Trenouth*}

Consultant Orthodontist (Retired), Royal Preston Hospital, Sharoe Green Lane, United Kingdom

*Corresponding author: Michael John Trenouth, Consultant Orthodontist (Retired), Royal Preston Hospital, Sharoe Green Lane, Preston, PR2 9HT, United Kingdom, Tel: +44/15395-3378; E-mail: michaeltrenouth@hotmail.co.uk

Received: 03 Jul, 2021 | Accepted: 24 Jul, 2021 | Published: 31 Jul, 2021

Citation: Trenouth MJ (2021) Anatomical and Centroid Point Variation in Cephalometrics. Int J Dent Oral Health 7(5): dx.doi.org/10.16966/23787090.375

Copyright: (C) 2021 Trenouth MJ. This is an open-access article distributed under the terms of the Creative Commons Attribution License, which permits unrestricted use, distribution, and reproduction in any medium, provided the original author and source are credited.

\section{Abstract}

Objective: The variance of cephalometric points depends not only on the variability of the points themselves but on the magnitude of the distances between them. The study was undertaken to test the effect of standardizing the variance by dividing it by the mean distance to give the standardized variance.

Materials and Methods: 5 centroid points and 18 anatomical points were located on 60 tracings of lateral cephalometric radiographs. The distances between each point and every other combination of points were measured. The mean distance, mean variance and standardized variance was calculated for each pair of points.

Results: Regression analysis was performed for each combination of mean distance, mean variance and mean standardized variance. The mean distance accounted for $33 \%$ of the variation in variance but only $2 \%$ of the variation in standardized variance.

Conclusions: Standardizing the variance virtually eliminated the effect of distance on variance. Centroid points were consistently less variable than anatomical points.

Keywords: Cephalometrics; Anatomical points; Centroid points; Variance; Regression analysis; Reference planes

\section{Introduction}

When successive tracings or radiographs are superimposed, there must be a reference system common to all records to make valid comparisons. The ultimate biological correctness of any description of growth or treatment outcome will depend on the stability of the reference points chosen. If growth is described by the changes of all other structures relative to a given set of reference points, then an arbitrary selection of another set of reference points will lead to still another description of growth.

Johnson introduced centroid analysis in 1960 [1] by finding the centre of area of an irregularly shaped outline. This was originally derived by cutting out the outline in cardboard and suspending it from two separate points and drawing in a vertical line. Where the two lines intersect is the centre of gravity or centre of area of a shape of uniform weight. Computer programs are now available to perform this operation. Centre of gravity was the invention of Archimedes [2]. This is a single point where the average weight or gravity acts coinciding with the geometrical centre, which is the point of balance. The centroid is based upon the law of moments. Newton used the centroid concept in the formulation of his laws of motion and universal gravitation [3].

The centroid or centre of area of an image represents the mean point within a shape about which it varies in space. Like other means, centroid points are subject to least variation relative to non-mean anatomical points. In the same way the distribution of the means of samples of a population shows less variation than the distribution of the individual values, that is, the standard error is always less than the standard deviation, figure 1 . The centroid of the whole skull outline lies on the same plane as the centroids of the cranium and face components divided along the cranial base to form the craniofacial centroid plane. This forms a more stable reference plane than the sella-nasion plane or any other plane based on anatomical points [1].

Evidence that centroid points are less variable than anatomical points has been derived by comparing the variation of angles and distances between every combination of pairs of defined lines and points, both anatomical and centroid. The mean variance can then be calculated for each of the angles and points measured. Centroid angles and points come out with the least variance in such studies [4-8].

Pairs of points are often correlated with each other since they are anatomically interdependent. This was described as topographic correlation by Solow B [9]. Topographical correlation can also be reduced by using polar co-ordinates from the craniofacial centroid to measure circular variance [10]. However, the variance will not only depend on the variability of the points between which distances are measured and also the magnitude of such distances. Variance is 
influenced by the distance between the points as well as their intrinsic variability. By compensating for the distance between the points this confounding factor is virtually eliminated providing a more valid measure of standardized variance. To eliminate the influence of different values for distance between points the variance was divided by the mean distance to give the standardized variance. No one has previously standardized the variance for differing distance between the points. For this reason the following study was undertaken to investigate the effect of standardizing for magnitude of distance. This method validates the stability of centroid points as a reference system to study craniofacial growth. Where such centroid points do move during growth this indicates a highly significant change.

\section{Materials and Methods}

A retrospective analysis was performed on 60 lateral cephalometric radiographs. These were taken on standard lateral cephalometry equipment with a 5 feet tube film distance and the central axis of the $\mathrm{x}$-ray beam in line with the cephalostat ear posts. The patients were orientated with the Frankfort plane horizontal. The cephalometric radiographs were all taken at the Department of Orthodontics, Royal Preston Hospital, Preston, England, on Siemens equipment. Cephalometric tracings were taken from the radiographs and scanned into a laptop computer. The tracings were performed manually using a sharp pencil on acetate paper over an illuminated opal light box. Centroid analysis was performed by the Autoceph program specially written for this task. This was developed by Roger Wordsworth of the Information Technology Department of the Royal Preston Hospital.

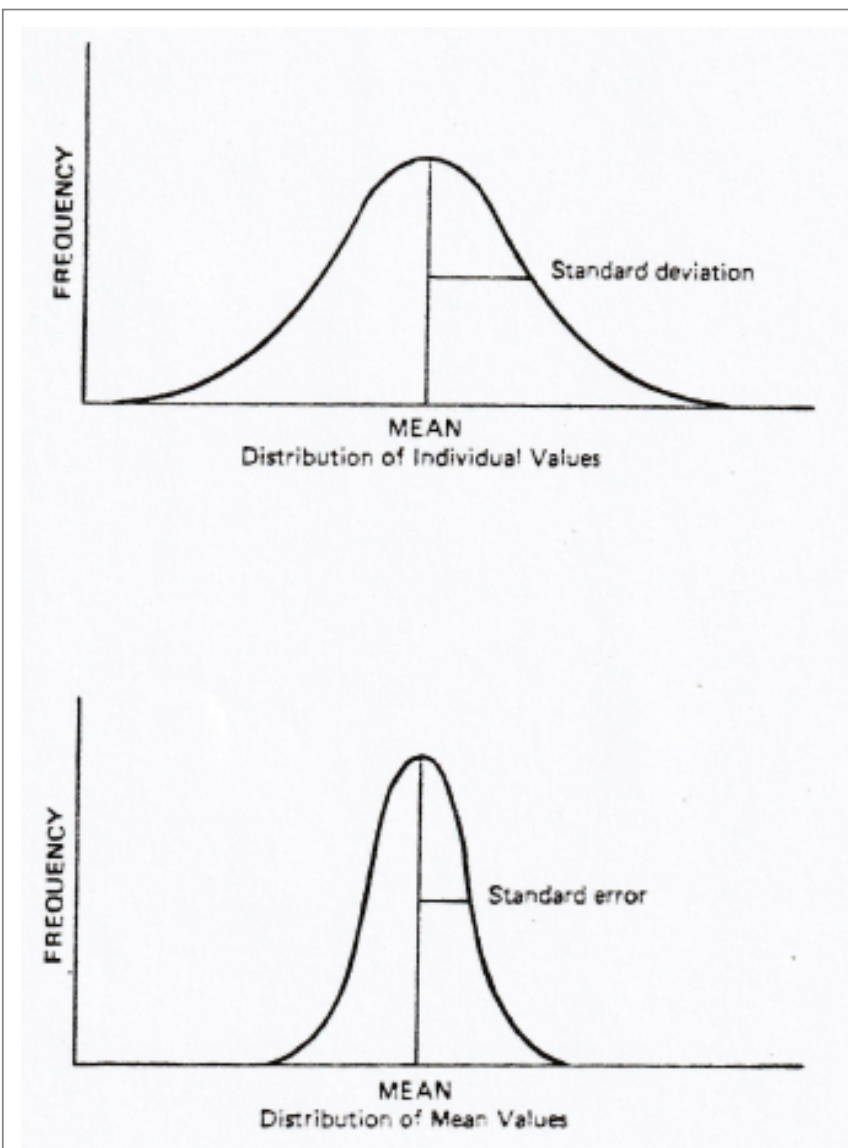

Figure 1: The normal distribution about the mean for individual values and mean values.
The software provides standard ceohalometric analysis by defining points, planes and angles. In addition centroid analysis was performed by finding the centre of area of a defined outline. It was also capable of measuring the distance between any two specified points.

The following anatomical points were located: figure 2. S (Sella), N (Nasion), ANS (Anterior Nasal Spine), PNS (Posterior Nasal Spine), A (Subspinale), B (Supramentale), UIE (Upper Incisal Edge), UIA (Upper Incisal Apex), LIE (Lower Incisal Edge), LIA (Lower Incisal Apex), Me (Menton), Go (Gonion), Ba (Basion), Oca (Occipital condyle apex), Eop (External Occipital Protuberance), La (Lambda), Br (Bregma), Gl (Glabella).

The following centroid points were generated from outlines of the total skull and cranium and face components: figure 2 CFC (Cranio Facial Centroid), CC (Cranial Centroid), FC (Facial Centroid), UFC (Upper Facial Centroid), LFC (Lower Facial Centroid).

The centroid of the cranium, face and total skull outline all lie on a straight line, the craniofacial centroid plane.

The following angles were measured relative to the craniofacial centroid plane: FCL (Facial Centroid Plane; line joining UFC to LFC), A-B (Dental Base), UIE-UIA (Upper incisor long axis), LIELIA (Lower incisor long axis), Go-Me (Mandibular plane), ANS-PNS (Maxillary plane), S-N (Sella Nasion plane).

The distances between each point and every other combination of points were measured. This was performed for both the 18 anatomical and 5 centroid points. For example CFC-CC, CFC-FC, CFC-UFC, CFC-LFC, CFC-S, etc: Followed by CC-FC, CC-UFC, CC-LFC, CC-S, etc: FC-UFC, FC-LFC, FC-S, FC-N, etc: and so on until all the possible combinations were exhausted. This was performed for each of the 60 radiographs. The mean distance, mean variance and standardized variance was calculated for the 60 values for each pair of points.

All linear measurements were corrected for magnification by the Autoceph program using the millimeter scale on the cephalograms for each individual patient. For valid comparisons it is important to correct the magnification as these vary between different studies and even between individual patients within the same study [11].

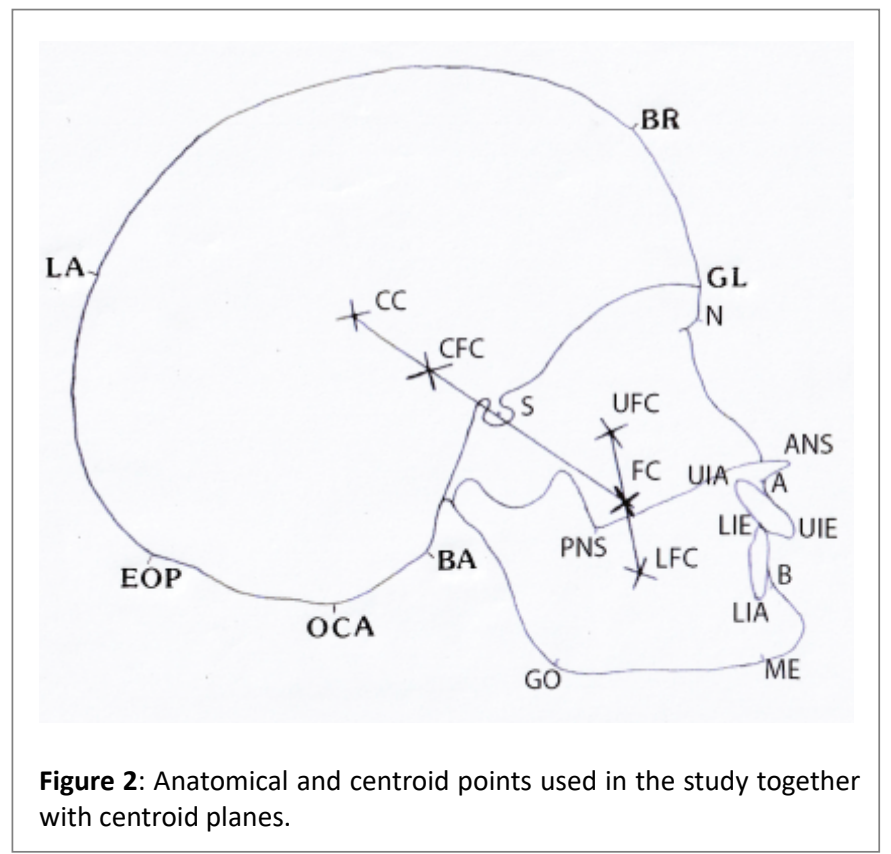




\section{Results}

The mean variance for the angular measurements of the various planes relative to the craniofacial centroid plane is shown in table 1 . The facial centroid plane had the lowest variance and was ranked first. The second least variable was the Sella Nasion plane and the incisor inclinations demonstrated the greatest variation.

The 5 centroid points were calculated and the 18 anatomical points were located. The distance between every combination of anatomical and centroid points was measured by the Autoceph program. The mean distances and variance was calculated for each combination of points for the 60 radiographs. The mean variance was standardized by dividing it by the mean distance and multiplying by 100 to give the mean standardized variance.

A linear regression model was used for the parameters mean distance and variance as well as mean distance and standardized variance and also variance and standardized variance. The results of regression analysis for each combination of mean distance, mean variance and mean standardized variance, are shown in table 2. It can be concluded that mean distance accounts for $33 \%$ of the variation in variance but only $2 \%$ of the variation in standardized variance. Hence standardizing the variance virtually eliminated the effect of mean distance on variance. However, the standardized variance still accounts for $65 \%$ of the variation in variance and is therefore a representative measure of this.

The mean standardized variance for all the distances between each set of two anatomical points was calculated as was the mean standardized variance between each set of anatomical and centroid points and each set of two centroid points, figure 3. It can clearly be seen that centroid to centroid point distances have the least variance followed by centroid to anatomical point distances, the anatomical to anatomical point distances having the greatest variance. Standardized variance is a more valid and reliable method of establishing that centroid points provide a more stable reference system.

Table 1: Mean variance cephalometric angles relative to craniofacial plane.

\begin{tabular}{|l|c|c|}
\hline \multicolumn{1}{|c|}{ Plane } & Mean Variance & Rank \\
\hline FCL & 4.88 & 1 \\
\hline N-S & 8.13 & 2 \\
\hline ANS-PNS & 13.00 & 3 \\
\hline A-B & 23.59 & 4 \\
\hline Go-Me & 45.87 & 5 \\
\hline UIE-UIA & 57.07 & 6 \\
\hline LIE-LIA & 67.49 & 7 \\
\hline
\end{tabular}

Table 2: Regression analysis.

\begin{tabular}{|l|c|c|c|}
\hline \multicolumn{1}{|c|}{$\mathbf{r}^{\mathbf{2}}$} & Mean Distance & Mean Variance & $\begin{array}{c}\text { Mean Standardised } \\
\text { Variance }\end{array}$ \\
\hline Mean Distance & --- & --- & --- \\
\hline Mean Variance & 0.33 & --- & --- \\
\hline $\begin{array}{l}\text { Mean } \\
\text { Standardised } \\
\text { Variance }\end{array}$ & 0.02 & 0.65 & --- \\
\hline
\end{tabular}

The mean variance for each individual point was calculated by adding the variance for all the distances between one particular point and all the other remaining anatomical and centroid points. The total variance was then divided by the total number of points (23) to give the mean variance for a particular point. The process was repeated for each remaining anatomical and centroid point providing each individual point with a mean value for mean variance, table 3 . The exact same procedure was repeated for standardized variance, table 4 .

The mean variance for the 5 centroid points (18.8 CI 16.4-21.1) was less than that of the 18 anatomical points (37.4 CI 26.2-48.7). The difference was statistically significant using a Mann Whitney U test $(\mathrm{p}<0.001)$, and the confidence intervals did not overlap.

The mean standardized variance for the 5 centroid points (23.1 CI 20.2-25.9) was again less than that of the 18 anatomical points (40.0 CI 38.4-41.5). The difference was statistically significant using a Mann Whitney $\mathrm{U}$ test $(\mathrm{p}<0.001)$, and the confidence intervals did not overlap.

\section{Discussion}

The present study clearly demonstrates that centroid points are less variable than anatomical points both in terms of angular and linear measurements. This finding is consistent with the results of previous studies. The exact order of variance seems to differ between different studies or even the same study on different data sets $[5,10]$. This is believed to be due to correlations between anatomical points which can modify the actual order of hierarchy. In theory the centroid of the total skull (craniofacial) should show the least variance because this represents the largest area. This should be followed by the cranium, face, lower face and upper face in terms of decreasing area and increasing variance. The results of the present study compared favourably to the only previously published state of the art methods $[5,10]$.

Mean distance accounted for $33 \%$ of the variation of the variance but only $2 \%$ of the variation in standardized variance. Hence standardizing the variance virtually eliminated the effect of varying distance between points on the variance. Standardizing the variance

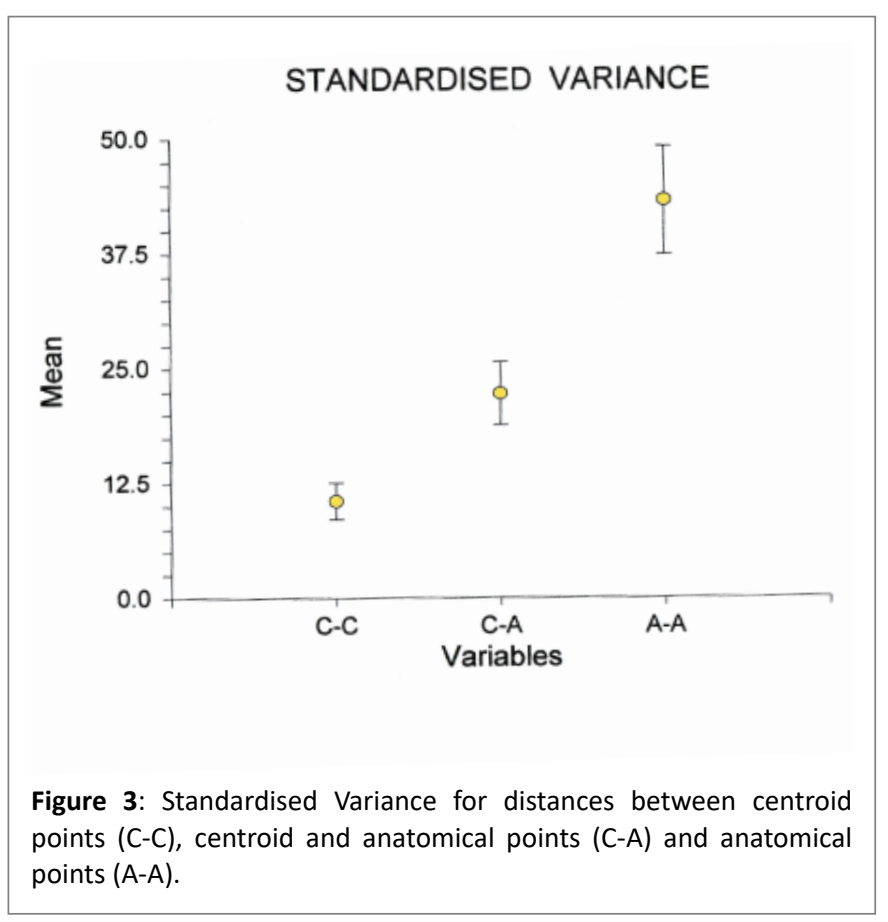


Table 3: Mean variance.

\begin{tabular}{|c|c|c|}
\hline Point & Mean & Rank \\
\hline CFC & 15.65 & 1 \\
\hline $\mathrm{CC}$ & 21.60 & 5 \\
\hline FC & 17.74 & 3 \\
\hline UFC & 17.25 & 2 \\
\hline LFC & 21.62 & 6 \\
\hline$S$ & 21.97 & 7 \\
\hline $\mathrm{N}$ & 28.24 & 13 \\
\hline ANS & 26.11 & 10 \\
\hline PNS & 21.23 & 4 \\
\hline A & 24.43 & 9 \\
\hline B & 27.99 & 12 \\
\hline UIE & 26.58 & 11 \\
\hline UIA & 23.48 & 8 \\
\hline LIE & 31.39 & 17 \\
\hline LIA & 29.44 & 14 \\
\hline $\mathrm{Me}$ & 38.74 & 19 \\
\hline Go & 47.87 & 21 \\
\hline $\mathrm{Ba}$ & 29.68 & 15 \\
\hline Oca & 36.49 & 18 \\
\hline Eop & 126.03 & 23 \\
\hline La & 60.81 & 22 \\
\hline $\mathrm{Br}$ & 43.27 & 20 \\
\hline GI & 30.06 & 16 \\
\hline
\end{tabular}

improved the hierarchical order of variance but did not completely achieve the ideal theoretical order, table 4 . Standardizing the variance obtained a better estimation of true variance because the confounding influence of differing distances between the points measured was largely eliminated.

In the present study, table 1 the facial centroid plane (FCL) showed least variance followed by S-N, ANS-PNS, A-B, Go-Me, UIE-UIA, LIE-LIA. This was exactly the same order as in the only previous study by Johnson \& Hubbold [5] on their main data set of 207 subjects (Table 3 of their study) but different from their pilot study on 33 subjects (Table 2 of their study).

In all the various studies, centroid planes were least variable; the incisor long axes were most variable with other reference planes somewhere between the two. The S-N plane was more variable than the CFC but was second least variable compared to other anatomical planes measured. S-N is one of the most commonly used reference planes in cephalometric analysis. You QL, Hagg U [12] showed that Panchertz's method of superimposition on S-N at $\mathrm{S}$ had a higher coefficient of reliability than either Bjorks structural or Ricketts fourposition methods. The present study clearly demonstrates that centroid points are superior reference points in cephalometric analysis because they have least variance compared to anatomical points.

Centroid analysis has been used to study craniofacial growth. Trenouth MJ [13] applied centroid analysis to the mean outlines of the skull from 1 to 18 years derived from the Bolton growth study. The Reference profile angle between the craniofacial centroid plane and
Table 4: Mean standardized variance.

\begin{tabular}{|c|c|c|}
\hline Point & Mean & Rank \\
\hline CFC & 19.18 & 1 \\
\hline $\mathrm{CC}$ & 20.71 & 2 \\
\hline FC & 24.75 & 4 \\
\hline UFC & 23.21 & 3 \\
\hline LFC & 27.43 & 5 \\
\hline$S$ & 29.21 & 6 \\
\hline $\mathrm{N}$ & 33.81 & 14 \\
\hline ANS & 33.16 & 12 \\
\hline PNS & 30.22 & 8 \\
\hline A & 31.60 & 10 \\
\hline B & 32.65 & 11 \\
\hline UIE & 31.60 & 10 \\
\hline UIA & 31.34 & 9 \\
\hline LIE & 33.95 & 16 \\
\hline LIA & 33.22 & 13 \\
\hline $\mathrm{Me}$ & 37.99 & 17 \\
\hline Go & 54.78 & 21 \\
\hline $\mathrm{Ba}$ & 40.76 & 18 \\
\hline Oca & 47.47 & 20 \\
\hline Eop & 111.01 & 22 \\
\hline La & 41.00 & 19 \\
\hline $\mathrm{Br}$ & 30.11 & 7 \\
\hline GI & 35.22 & 15 \\
\hline
\end{tabular}

facial centroid plane was found to decrease during growth which is associated with bimaxillary prognathism $[14,15]$. The centroid of the lower face moves forwards relative to the other centroids indicating prolonged growth of the lower face beyond that of the upper face. As yet no growth prediction studies have been performed using centroid points.

Trenouth MJ [16] also used centroid analysis to study prenatal growth. The centroid of the upper face moved forward relative to the lower face, indicating forward growth of the maxilla in excess of much smaller growth of the mandible. The protrusion of the maxilla was produced by flattening out of the cranial base due to brain growth [17].

Trenouth MJ [8] used centroid analysis to evaluate treatment outcome for the Twin block appliance. The reference profile angle decreased indicating an improvement in the dental base relationship. There was forward movement of the lower face centroid but not the upper face centroid which was unchanged, indicating forward movement of the mandible. This was further confirmed by statistically significant increases in the distances $\mathrm{CFC}$ to point $\mathrm{B}$, Menton and lower incisor apex and edge. The upper incisors retroclined with a statistically significant decrease in the distance CFC to upper incisor edge, the apex remaining unchanged.

Mackay F, et al., [18] applied centroid analysis to adults with class III malocclusions prior to orthognathic surgery. Cluster analysis produced 5 subgroups. All subjects had a degree of mandibular prognathism while $14 \%$ had maxillary retrognathism and $58 \%$ increased lower face height. Centroid analysis has also been used to 
compare facial morphology of Chinese, Indonesians and Europeans [19]. Class III skeletal pattern was more prevalent in the Chinese, Class II in Europeans, whilst Indonesians were predominantly Class I with bimaxillary prognathism. Centroid based methods have even been used to investigate palaeolithic hand axes and Egyption pottery [20].

Centrographic analysis, introduced by Fishman, [21] also uses centroids but these are centres of a geometric form usually a triangle. They are derived by connecting the triangular vertex to the midpoint of the opposite side in at least two instances, where the two lines intersect is the centre of area of the triangle or centrographic centroid. This method is simpler to perform because it only requires the marking of a minimum of 4 points $(\mathrm{Ba}, \mathrm{Na}, \mathrm{Gn}, \mathrm{A})$. It is highly likely that centrographic points are less variable than anatomical points but no variance studies have been published. More recently various applications of this method has been used by Dolce C et al., [22]; Murata S, et al., [23]; Murata S [24]; Al-Shahrani IS, et al., [25]; Nehete $\mathrm{AB}$ and Hazare PV [26]; Yagci A, et al., [27]; Jayapal J, et al., [28].

Abraham RA, Sundareswaran S [29] compared Rickets, Pancherz and Centrographic methods of superimposition and found all three to be equally reliable. However centrographic analysis provided more stable reference points. During Twin block treatment the Facial centroid axis maintained a relatively stable special position. Forward movement of the lower facial centroid occurred in all cases treated with the Twin block appliance. This finding indicated a true mandibular skeletal change, confirming the finding of Trenouth MJ [8]. By using centroid analysis it was possible to locate the exact sites where treatment changes occurred because of the stability of Facial centroid reference axis.

\section{Summary and Conclusions}

- Centroid points are less variable than anatomical points.

- Standardising the variance virtually eliminates the effect of varying distance between points on the variance.

- The craniofacial centroid plane provides a stable reference structure from which to measure changes during growth and treatment.

\section{Acknowledgements}

I should like to thank Mr. Roger Wordsworth for writing and developing the AutoCeph program for centroid analysis without which this project could not have been undertaken. I should also like to thank Dr. J.S. Johnson for teaching me the principles of centroid analysis.

\section{References}

1. Johnson JS (1960) The use of centres of gravity in cephalometric analysis. Dent Pract Dent Rec 10: 107-113.

2. Netz R, Noel W (2007) The Archimedes Codex, Weidenfield \& Nicholson, London.

3. Pask C (2013) Magnificent Principia. Prometheus Books, New York, USA.

4. Johnson JS (1972) A statistical survey, illustrating the uses of mathematical centres in the analysis of the human lateral skull cephalostat radiograph. London University.

5. Johnson JS, Hubbold RJ (1982) An introduction to centroid cephalometrics. Br J Orthod 9: 32-36.

6. Trenouth MJ (1989) The stability of anatomical and centroid reference points in cephalometric analysis. Angle Orthod 59: 283290.
7. Trenouth MJ (1993) Variation of anatomical and centroid points in the human fetal skull. Angle Orthod 63: 39-44.

8. Trenouth MJ (2006) Centroid analysis of twin-block appliance treatment for class II division 1 malocclusion. World J Orthod 7: 159-164.

9. Solow B (1966) The pattern of craniofacial associations: A morphological and methodological correlation and factor analysis study on young adults. Acta Odont Scand.

10. Johnson JS, Laycock PJ (1980) The use of circular variances in aiding cephalometric orthodontic analysis. IRCS Med Sci 8: 164-173.

11. Dibbetts JMH, Nolte K (2002) Effects of magnification on lateral cephalometric studies. Am J Orthod Dentofacial Orthop 122: 196201.

12. You QL, Hagg U (1999) A comparison of three superimposition methods. Eur J Orthod 21: 717-725.

13. Trenouth MJ (2008) Area centroid analysis of craniofacial growth. Cranio-view 17: 25-31.

14. Johnson JS (1978) A new approach to cephalometric analysis of the dental base relationship. Angle Orthod 48: 23-32.

15. Johnson JS, Eid AA (1979) Recent developments in diagnosis and treatment planning of antero-posterior jaw discrepancies from the lateral skull cephalostat radiograph. Br J Oral Maxillofac Surg 17: 256-264.

16. Trenouth MJ (1981) Angular changes in cephalometric and centroid planes during foetal growth. Br J Orthod 8: 77-81.

17. Trenouth MJ (1984) Shape changes during human fetal craniofacial growth. J Anat 139: 639-651.

18. Mackay F, Jones JAH, Thompson R, Simpson W (1992) Craniofacial form in Class III cases. Br J Orthod 19: 15-20.

19. Johnson JS, Soetamat A, Winoto NS (1978) A comparison of some features of the Indonesian occlusion with those of two other ethnic groups. Br J Orthod 5: 183-188.

20. Tyldesley JA, Johnson JS, Snape SR (1985) "Shape" in archaeological artifacts: two case studies using a new analytical method. Oxford J Archael 4: 19-30.

21. Fishman LS (1997) Individualised evaluation of facial form. Am J Orthod Dentofacial Orthop 11: 510-517.

22. Dolce C, Schader RE, McGorray SP, Wheeler TT (2005) Centrographic analysis of 1-phase versus 2-phase treatment for class II malocclusion. Am J Orthod Dentofacial Orthop 128: 195-200.

23. Murata S, Nakamura S, Nagahara K (2006) Evaluation of the centroid method of occlusion for studying mandibular and maxillary growth. Eur J Orthod 28: 345-351.

24. Murata S (2007) Use of the centroid method of occlusion for studying the vertical and horizontal relationship of the mandible and maxilla. Eur J Orthod 29: 600-604.

25. Al-Shahrani IS, Al-Balkhi KM, Al-Madi A (2010) The applicability of a non-anatomical soft and hard tissue centroid line (S\&H centroid) in cephalometrics. Saudi Dent J 22: 63-67.

26. Nehete AB, Hazare PV (2012) An evaluation of centrographic analysis as compared with conventional cephalometric analysis. J Ind Orthod Soc 46: 38-42.

27. Yagci A, Ramoglu SI, Usal T, Karaman Al, Ozdiler E (2013) Standards of centrographic analysis in an Anatolian Turkish population. Turkish J Orthod 26: 36-44. 
28. Jayapal J, Venkatasubramanian $P$, Sundararajan $S$, Parameswaran R, Vijayalakshmi D (2020) Assessment of treatment outcomes using centrographic analysis of skeletal class II malocclusion. Saudi J Oral Dent Res 5: 24-29.

29. Abraham RA, Sundareswaran S (2019) Comparison of Rickets, Pancherz and Centrographic superimposition methods in posttreatment assessment of the effects of Twin block-appliance therapy. Iran J Ortho 13: e12512. 